\title{
How to Design Optimal Accelerated rTMS Protocols Capable of Promoting Therapeutically Beneficial Metaplasticity
}

\author{
Alix C. Thomson ${ }^{1,2,3}$ and Alexander T. Sack ${ }^{1,2,3 *}$ \\ ${ }^{1}$ Department of Cognitive Neuroscience, Faculty of Psychology and Neuroscience, Maastricht University, Maastricht, \\ Netherlands, ${ }^{2}$ Department of Psychiatry and Neuropsychology, School for Mental Health and Neuroscience (MHeNS), \\ Maastricht, Netherlands, ${ }^{3}$ Centre for Integrative Neuroscience, Faculty of Psychology and Neuroscience, Faculty of Health, \\ Medicine and Life Sciences, Maastricht University, Maastricht, Netherlands
}

Keywords: metaplasticity, homeostatic plasticity, hebbian plasticity, transcrancial magnetic stimulation (TMS), accelerated rTMS

\section{INTRODUCTION}

OPEN ACCESS

Edited by:

Mariagiovanna Cantone,

Sant'Elia Hospital, Italy

Reviewed by:

Paolo Maria Rossini, Catholic University of the Sacred Heart, Italy

Wickliffe C. Abraham,

University of Otago, New Zealand

*Correspondence:

Alexander T. Sack a.sack@maastrichtuniversity.nl

Specialty section:

This article was submitted to

Neurorehabilitation,

a section of the journal

Frontiers in Neurology

Received: 28 August 2020 Accepted: 22 October 2020

Published: 05 November 2020

Citation:

Thomson AC and Sack AT (2020) How to Design Optimal Accelerated rTMS Protocols Capable of Promoting

Therapeutically Beneficial Metaplasticity.

Front. Neurol. 11:599918.

doi: 10.3389/fneur.2020.599918
Our brain is comprised of billions of neurons, which can connect via synapses that rely on electrical signaling and the release of chemical messengers to communicate and propagate signals through neural networks. By forming such networks, neurons are capable of monitoring previous firing activity, and using this information to adapt subsequent firing rate. This so-called activitydependent plasticity is critical for the encoding of new information, and the tuning of (low activity) connections (1-3). The physiological mechanisms of synaptic plasticity have largely been attributed to Long-Term Potentiation (LTP) $(4,5)$, and Long-Term Depression (LTD) (6-8), which result from molecular processes such as receptor trafficking or synaptic scaling (3). Both LTP and LTD are induced by postsynaptic NMDA receptor activation, which lead to an influx of calcium into the postsynaptic dendrites (8-10). This triggers a complex series of intracellular signaling cascades, resulting in synaptic modifications such as AMPA receptor trafficking $(11,12)$. The pattern of stimuli delivered to the post synapse determines whether LTP or LTD will occur; low frequency stimulation induces LTD, whereas high frequency stimulation induces LTP $(8,13)$. These processes underlie much of our knowledge on the molecular mechanisms of learning and memory.

However, if the principles of Hebbian synaptic plasticity (LTP, LTD) alone were to drive the strengthening and weakening of synaptic connections, activity would, over time, be driven toward destabilization. This is because continuously firing synapses could only become stronger (driven to saturation) and unused synapses quiescent (until completely lost) (14). Consider a synapse that is strengthened by LTP; meaning the presynaptic neuron becomes more effective at depolarizing the postsynaptic neuron. With each continued stimulation, the postsynaptic neuron will be more easily depolarized, in a positive feedback loop, resulting in a hyperexcitable postsynaptic neuron. Over time, not only will the original presynaptic connection be strengthened, but other unrelated presynaptic inputs could cause a depolarization of the hyperexcitable postsynaptic neuron, resulting in unregulated synaptic transmission (15). Therefore, other mechanisms must exist, which regulate synaptic plasticity on a global network level to maintain stability of synapses and maintain specificity of neural activity $(16,17)$.

Metaplasticity refers to any change in the direction or degree of synaptic plasticity (ex. LTP, LTD) based on prior neural activity (18). While both synaptic and metaplasticity are dependent on previous neural activity, metaplasticity does not directly alter the efficacy of synaptic transmission 
(as LTP/LTD), but it adjusts the neurons' ability to induce LTP/LTD with subsequent neural activity. Metaplasticity in some sense can be considered as the plasticity of synaptic plasticity, e.g., maintaining the dynamic nature of a neuron's firing threshold, when this neuron reaches a certain firing rate $(16,18,19)$. Metaplasticity works through similar synaptic modifications as LTP/LTD, such as NMDA receptor activation and modification (20), and changes in calcium signaling triggering complex signaling cascades (18). Metaplastic modifications, for example at NMDA receptors, can occur either at specific synapses or across the whole neuron, and on time scales from minutes to weeks (19). Depending on the temporal pattern and strength of previous neural activity, metaplastic mechanisms can be additive; for example promoting increased synaptic strengthening through repeated excitatory (LTPinducing) stimulation. Metaplasticity can also be stabilizing; for example acting against subsequent synaptic strengthening when repeating excitatory (LTP-inducing) stimulation $(19,21)$. This stabilizing form of metaplasticity is often referred to as homeostatic metaplasticity, as it specifically regulates the dynamic threshold of synaptic plasticity to maintain equilibrium, or homeostasis $(16,17)$. We hypothesize, based on research from human and animal studies, that the timing between excitatory stimulations are what differentiate between promoting additive or homeostatic metaplasticity.

We focus on the role of metaplasticity in Transcranial Magnetic Stimulation (TMS). We describe the recent use of accelerated (repeated) stimulation protocols, both in research and clinical applications, and the molecular mechanisms required to promote either homeostatic or additive metaplastic effects. Finally, we showcase the therapeutic potential of accelerated stimulation, and hypothesize that increasing the currently practiced stimulation intervals may be more efficacious in promoting additive metaplastic effects in various clinical applications of rTMS in rehabilitation, neurology, psychiatry, and cognitive decline.

\section{METAPLASTICITY IN TMS}

TMS is a widespread and increasingly popular non-invasive brain stimulation technique, where electromagnetic pulses allow stimulation to pass non-invasively through the skull (22). When pulses are applied in a certain pattern, as repetitive TMS (rTMS), protocols can have lasting excitatory or inhibitory effects (23-25). Two commonly used stimulation protocols are intermittent Theta Burst Stimulation (iTBS), requiring only $3 \mathrm{~min}$ of stimulation time, resulting in a lasting increase of cortical excitability, and continuous Theta Burst Stimulation (cTBS), requiring only $40 \mathrm{~s}$ of stimulation for a lasting decrease in cortical excitability (26). The after effects of these protocols have been shown for up to $1 \mathrm{~h}$ following stimulation $(26,27)$.

While iTBS is normally an excitatory protocol, causing an increase in cortical excitability of the stimulated brain region, it has been shown that when applied twice in quick succession iTBS effects switch from excitatory to inhibitory (28). Conversely, when cTBS (an inhibitory protocol) is applied for double the normal duration, its effects switch from inhibitory to excitatory (28). Several studies have reported similar effects of repeating iTBS or cTBS stimulation protocols, with the timing between protocols being an important factor in the magnitude and direction of aftereffects $(19,29,30)$. For example, using a "priming" iTBS protocol which does not induce plasticity, followed by a "test" iTBS protocol has shown that short intervals of $5 \mathrm{~min}$ between priming and test resulted in homeostaticlike changes in excitability, i.e., an opposite effect. Interestingly, longer breaks of 15 min resulted in an increase in MEP amplitude after the test iTBS (30). However, $15 \mathrm{~min}$ between priming and test iTBS/cTBS has also been shown to induce in homeostaticlike metaplastic effects (29). While the timing between repeated TBS sessions is clearly important, the optimal interval is less clear. 15 min between iTBS sessions has been shown to promote both homeostatic (29) and MEP enhancement after the second iTBS (30), while $10 \mathrm{~min}$ between priming and test iTBS has shown enhancement of MEP amplitude (31), but 5 and 20 min between iTBS sessions did not (32). Therefore, when 2 iTBS sessions are repeated with short ( $<30 \mathrm{~min}$ ) between, conflicting effects on MEP amplitude have been reported.

"Accelerated" protocols, which consist of multiple stimulation sessions on a single day, have recently been introduced for the treatment of depression (33-37). Due to their short duration, the TBS protocols, in particular iTBS, have been promising candidates for accelerated protocols (38). Also, a large trial recently found that iTBS was not-inferior to the classical $10 \mathrm{~Hz}$ rTMS protocol, confirming the clinical potential of this shorter stimulation protocol to treat depression (39). Indeed, several studies have shown additional benefits for accelerated iTBS protocols in the treatment of severe, treatment resistant depression $(40,41)$. In the clinic, an interval of $15 \mathrm{~min}$ is often used between iTBS sessions, with these sessions repeated up to 5 times on a single treatment day $(40,41)$.

We recently conducted a study investigating the effects of accelerated iTBS over motor cortex, consisting of 5 repeated iTBS sessions in a single day. iTBS with 8- or 15-min time interval between sessions were delivered to healthy participants in a fully within subject design; where participants received 4 different conditions (accelerated iTBS with 8-min intervals, accelerated iTBS with 15-min intervals, single iTBS and sham) (42). We compared change in Motor Evoked Potential (MEP) amplitude up to $90 \mathrm{~min}$ following stimulation, across the stimulation conditions.

We found that there was no difference in the effects of accelerated iTBS on MEP amplitude, also when compared to sham stimulation, and thus no additive metaplasticity induced by five stimulation sessions applied successively in 8 - or 15min intervals. We argue that such intervals between iTBS protocols are likely too short to avoid processes of homeostatic plasticity. With only 8 or $15 \mathrm{~min}$ between sessions, homeostatic mechanisms may be working against additive metaplastic effects to maintain network stability and therefore result in a net effect of no change in excitability following these accelerated protocols (42). 


\section{TIMING-DEPENDENT METAPLASTICITY}

In agreement with this notion, animal studies in rats, and rat hippocampal slices have shown that a sufficiently long pause between excitatory stimulation sessions was necessary for additive (LTP) plasticity effects to occur (43-45). This may have to do with the time required for metaplasticity mechanisms, for example synapse strengthening with AMPA receptor trafficking (15).

It has been well-established in animal studies, that a single round of TBS (a 4-pulse burst at $100 \mathrm{~Hz}$, repeated at $5 \mathrm{~Hz}$ for 10 bursts) is effective at inducing LTP in CA1 hippocampal pyramidal neurons $(46,47)$. TBS has since then been used extensively to reliably induce LTP in vitro (48). Interestingly, repeating this single TBS protocol with a time interval of $>40 \mathrm{~min}$, was capable of almost doubling the potentiation compared to the first TBS alone (43). This additional potentiation is thought to work through strengthening the smaller synapses which weren't strengthened by the first TBS protocol (43). This may have to do with the number of AMPA receptors; smaller synapses contain fewer AMPA receptors and therefore don't generate a response to trigger a depolarization following a single TBS (43). Several other studies have provided evidence for increased potentiation by spaced TBS, however the magnitude and duration of the effects depended on a series of factors such as rat strain, rat age, and the time interval. In adult Wistar rats, adult Long-Evans (LE) rats, and young LE rats, $4 \mathrm{~h}$ was required between TBS to induce additional potentiation $(44,45)$. However, in young Sprague Dawley (SD) rats, a single TBS repeated at $1-\mathrm{h}$ intervals could induce further potentiation, following up to 3 repeated TBS stimulations (4 did not produce additional potentiation) $(43,45)$. These different studies used different stimulation intensities; Frey et al. (44) found that reducing stimulation intensity in the second stimulation was effective for promoting potentiation 4h later, while Cao and Harris (45) and Kramár et al. (43) kept stimulation intensities constant. However, these studies consistently show that additional potentiation following repeated TBS in animal slices is possible. Enhanced, additive LTPlike plasticity may be promoted when repeating TBS with 50-60 min between sessions $(43,45)$. After 3 TBS protocols, spaced $60 \mathrm{~min}$ apart, potentiation had been raised to $150 \%$ baseline, which is about three times higher than if just one protocol was given $(43,48)$. This suggests that 3 TBS protocols repeated at $60 \mathrm{~min}$-intervals may be effective at promoting maximal, additive metaplasticity effects (Figure 1A). If there is less time between TBS protocols, for example $10 \mathrm{~min}$, homeostatic metaplasticity mechanisms may dominate, promoting a stabilizing rather than additive plasticity response (Figure 1B).

\section{DISCUSSION}

Activity-dependent metaplasticity is considered to be homeostatic if the first stimulation protocol alters the threshold for subsequent LTP/LTD in the opposite direction, thereby stabilizing (network) brain activity (49). Interestingly, this reversal of aftereffects has been shown specifically when stimulation protocols were given with a short $(0-5 \mathrm{~min})$ interval $(28,30)$, providing support for homeostatic metaplasticity mechanisms in rTMS protocols (19). While homeostatic metaplasticity mechanisms are important for stabilizing network activity, they can be counteractive when promoting plasticity effects through rTMS. In fact, when applying rTMS protocols, the explicit goal is not stabilization but promotion of additive, increased plasticity effects.

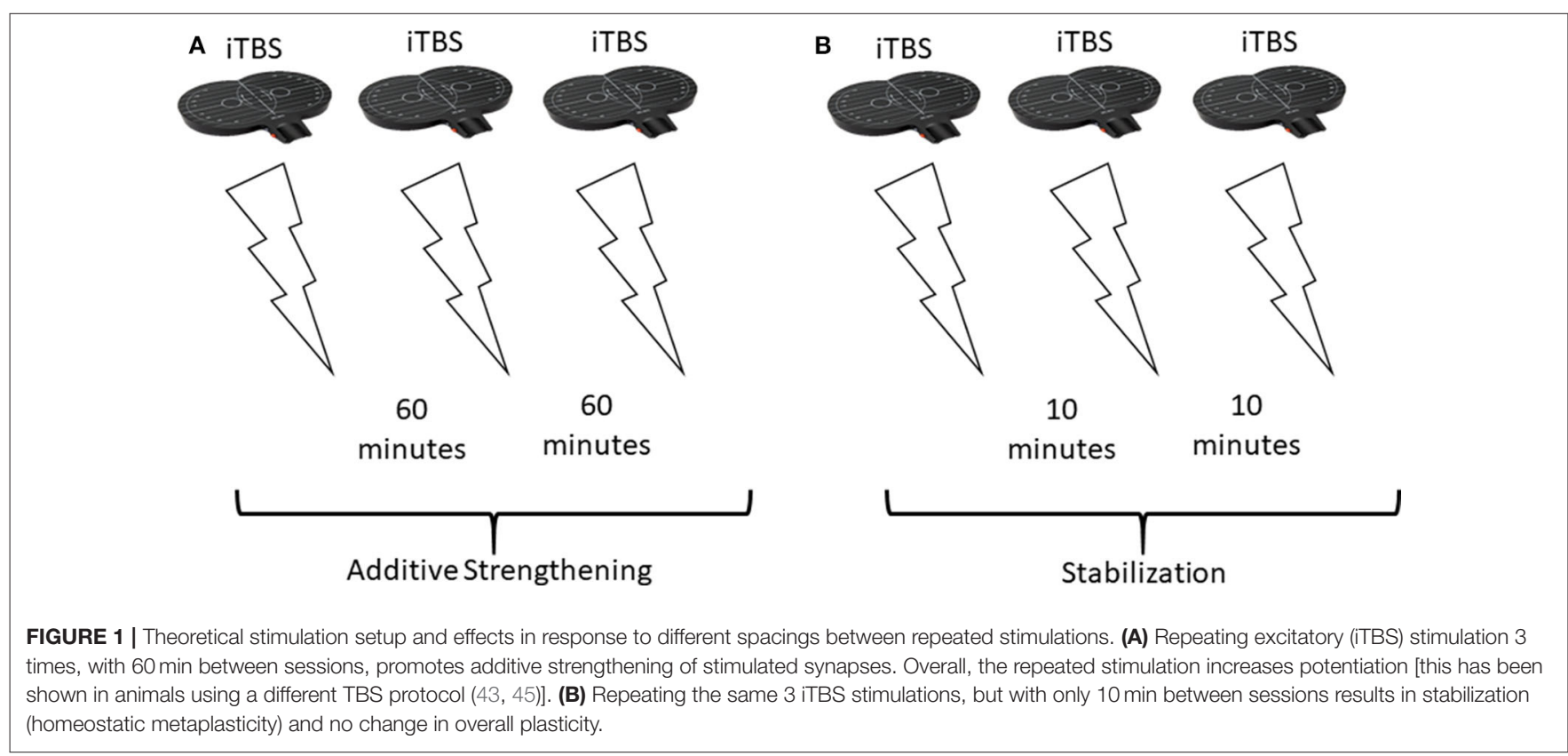


Animal studies have shown that timing is important in the molecular mechanisms underlying metaplasticity. While there is overlap between the mechanisms of additive and homeostatic metaplasticity, there are temporal differences which may differentiate between both principles at the molecular level. Based on evidence form animal models, leaving $60 \mathrm{~min}$ between excitatory stimulation protocols may promote additive rather than homeostatic metaplastic effects in accelerated TMS treatment protocols.

\section{Clinical Implications}

If longer intervals between iTBS sessions are capable of promoting additive metaplasticity, as has been shown in animal studies (43) as well as improving clinical outcomes in the treatment of depression (50), longer spaced intervals between iTBS sessions will likely be beneficial for other therapeutic applications of iTBS. iTBS is increasingly being used as a treatment in a range of clinical applications such as rehabilitation, as well as neurological and psychiatric disorders. For example, to promote motor recovery after stroke (51), for managing spasticity associated with Multiple Sclerosis (MS) (52), and decreasing obsessive symptomatology associated with Obsessive Compulsive Disorder (OCD) (53), just to name a few. These protocols all must adhere to the established safety guidelines (54), and recommendations for clinical TMS use $(55,56)$. These include total pulse number, interval between TBS session, intensity of stimulation, and cumulative weekly applications (54). Accelerated iTBS has been successfully and safely used in the treatment of depression $(38,40,41)$, with patients receiving a total of 32,400 pulses at $110 \%$ resting motor threshold, over 20 sessions ( 5 sessions per day, 15 min between sessions) in 4 days (41). Therefore, while following the established safety guidelines is the upmost priority, and local health authorities should always approve each stimulation protocol (54), delivering three iTBS sessions on a single day with $1 \mathrm{~h}$ between sessions should theoretically be safe and tolerable for most patients.

rTMS is also used as a treatment for the cognitive decline associated with neurodegenerative disorders such as dementia, and Alzheimer's Disease (AD) (57-61). However, there are ethical implications of using rTMS for cognitive enhancement, in particular in healthy participants (62). It is important to maintain the consensus ethical requirements that (1) participants/patients provide informed consent, (2) the benefit of the research outweigh the risks, and (3) there is equal distribution of burdens and benefits across patients (this is violated if a particular group of patients with different economic, physical or social conditions) (54).

Importantly, the here described principles of additive and homeostatic metaplasticity not only apply to the here discussed accelerated TMS treatments and the question of optimal time interval between its repeated stimulation sessions, but likewise can be used to explain and optimize other forms of plasticityinducing TMS protocols such as Paired Associated-Stimulation (PAS) or paired-coil TMS (pcTMS).

In humans, neural excitability and synaptic plasticity can be probed by TMS to peripheral nerves and motor cortex $(63,64)$.
In such a transcortical loop, timings of afferent (muscle/nerve to brain), cortical, and efferent (brain to muscle) responses can be used to quantify central motor excitability (63). For example, delivering a conditioning TMS pulse to an afferent tract (ex. the wrist), followed (10-48 ms) by stimulation of the efferent tract (motor cortex), will alter Motor Evoked Potentials (MEP's) measured from thumb flexor muscles (63). It has been shown that wrist stimulation $20-22 \mathrm{msec}$ preceding motor cortex stimulation elicits a facilitated MEP, with a latency of about $1 \mathrm{~ms}$, compared to MEPs given without the conditioning wrist stimulation (63). Repeating this afferent (wrist) efferent (motor cortex) stimulation, in Paired Associated Stimulation (PAS), can induce lasting effects on motor cortex excitability $(64,65)$, providing evidence for synaptic plasticity. Interestingly, evidence of homeostatic and additive metaplastic responses have also been recorded using PAS stimulation $(66,67)$. When two LTP-inducing PAS protocols were separated by $30 \mathrm{~min}$, a decrease in MEP amplitude was measured, indicating a homeostatic (stabilizing) metaplastic responses (66). Similarly, LTD-inducing PAS immediately preceding a motor-learning task facilitated motor-learning (67), again providing support for homeostatic plasticity mechanisms dominating at early time points following stimulation.

Additionally, the effects of brain stimulation are not only localized to the site of stimulation, but can also spread to different areas through complex cortical networks. Similarly to PAS, this has been shown using paired-coil TMS (pcTMS), where multiple coils are used to probe different cortical areas and assess connectivity $(68,69)$. For example, a single TMS pulse to motor cortex can cause a depression of the MEP measured following a subsequent $(6-30 \mathrm{~ms})$ TMS pulse to contralateral motor cortex (70). Therefore, TMS can also be used to assess connectivity between brain areas (68). In other words, TMS stimulation can propagate to different cortical regions, having both local and remote effects on (meta) plasticity. This has valuable clinical implications, where inducing plasticity effects in a cortical network are important (69). In stroke patients for example, localized damage can disrupt connectivity and can have functional consequences (69), therefore stimulation effects should promote network plasticity, rather than localized plasticity. Similarly, in the treatment of depression, superficial stimulation uses cortical connectivity to influence deeper cortical structures, resulting in improvement of clinical symptoms (71, 72). Therefore, it is important to use TMS to strengthen connectivity, and to promote additive, metaplastic changes also on the network activity level.

With the increasing and widespread application of rTMS protocols in the clinic, it is important to optimize protocols to maximize their effects, while remaining within established safety and ethical guidelines for use in the clinic $(54,56)$. Single iTBS has proven promising, but accelerated iTBS at longer time intervals $(60 \mathrm{~min}$ ) between sessions could maximize clinical outcomes through additive metaplasticity, preventing homeostatic metaplasticity from stabilizing stimulation effects. Clinical efficacy of PAS and pcTMS protocols may be similarly increased by optimizing the timing between stimulations according to these principles of metaplasticity. 


\section{AUTHOR CONTRIBUTIONS}

AS: conceptualization, writing-review and editing, supervision, and funding acquisition. AT: investigation, writing-original draft preparation, and visualization. All authors contributed to the article and approved the submitted version.

\section{REFERENCES}

1. Hebb DO. The Organization of Behaviour: A Neurophysiological Theory. New York, NY: Wiley. (1949).

2. Stent GS. A Physiological mechanism for hebb's postulate of learning. Proc Natl Acad Sci USA. (1973) 70:997-1001. doi: 10.1073/pnas.70.4.997

3. Turrigiano GG, Leslie KR, Desai NS, Rutherford LC, Nelson SB. Activitydependent scaling of quantal amplitude in neocortical neurons. Nature. (1998) 391:892-6. doi: 10.1038/36103

4. Madison DV, Malenka RC, Nicoll RA. Mechanisms underlying long-term potentiation of synaptic transmission. Annu Rev Neurosci. (1991) 14:37997. doi: 10.1146/annurev.ne.14.030191.002115

5. Bliss TV, Lomo T. Long-lasting potentiation of synaptic transmission in the dentate area of the anaesthetized rabbit following stimulation of the perforant path. J Physiol. (1973) 232:331-56. doi: 10.1113/jphysiol.1973.sp010273

6. Linden DJ, Connor JA. Long-term synaptic depression. Annu Rev Neurosci. (1995) 18:319-57. doi: 10.1146/annurev.ne.18.030195.001535

7. Ito M, Kano M. Long-lasting depression of parallel fiber-Purkinje cell transmission induced by conjunctive stimulation of parallel fibers and climbing fibers in the cerebellar cortex. Neurosci Lett. (1982) 33:2538. doi: 10.1016/0304-3940(82)90380-9

8. Dudek SM, Bear MF. Homosynaptic long-term depression in area CA1 of hippocampus and effects of N-methyl-D-aspartate receptor blockade. Proc Natl Acad Sci USA. (1992) 89:4363-7. doi: 10.1073/pnas.89.10.4363

9. Bliss TV, Collingridge GL. A synaptic model of memory: long-term potentiation in the hippocampus. Nature. (1993) 361:31-9. doi: 10.1038/361031a0

10. Dudek SM, Bear MF. Bidirectional long-term modification of synaptic effectiveness in the adult and immature hippocampus. J Neurosci. (1993) 13:2910-8. doi: 10.1523/JNEUROSCI.13-07-02910.1993

11. Malenka RC, Bear MF. LTP and LTD: an embarrassment of riches. Neuron. (2004) 44:5-21. doi: 10.1016/j.neuron.2004.09.012

12. Malenka RC, Nicoll RA. Long-term potentiation-a decade of progress? Science. (1999) 285:1870-4. doi: 10.1126/science.285.5435.1870

13. Huang YY, Colino A, Selig DK, Malenka RC. The influence of prior synaptic activity on the induction of long-term potentiation. Science. (1992) 255:7303. doi: $10.1126 /$ science. 1346729

14. Abbott LF, Nelson SB. Synaptic plasticity: taming the beast. Nat Neurosci. (2000) 3(Suppl:11)78-83. doi: 10.1038/81453

15. Turrigiano GG. The self-tuning neuron: synaptic scaling of excitatory synapses. Cell. (2008) 135:422-35. doi: 10.1016/j.cell.2008.10.008

16. Bienenstock EL, Cooper LN, Munro PW. Theory for the development of neuron selectivity: orientation specificity and binocular interaction in visual cortex. $J$ Neurosci. (1982) 2:32-48. doi: 10.1523/JNEUROSCI.02-01-00032.1982

17. Turrigiano GG, Nelson SB. Homeostatic plasticity in the developing nervous system. Nat Rev Neurosci. (2004) 5:97-107. doi: 10.1038/nrn1327

18. Abraham WC, Bear MF. Metaplasticity: the plasticity of synaptic plasticity. Trends Neurosci. (1996) 19:126-30. doi: 10.1016/S0166-2236(96)80018-X

19. Muller-Dahlhaus F, Ziemann U. Metaplasticity in human cortex. Neuroscientist. (2015) 21:185-202. doi: 10.1177/1073858414526645

20. Philpot BD, Cho KK, Bear MF. Obligatory role of NR2A for metaplasticity in visual cortex. Neuron. (2007) 53:495-502. doi: 10.1016/j.neuron.2007.01.027

21. Li J, Park E, Zhong LR, Chen L. Homeostatic synaptic plasticity as a metaplasticity mechanism - a molecular and cellular perspective. Curr Opin Neurobiol. (2019) 54:44-53. doi: 10.1016/j.conb.2018.08.010

\section{FUNDING}

This work was supported by the Netherlands Organization for Scientific Research (NWO, Vici to A.T.S 453-15-008), and an internal grant from the Centre for Integrative Neuroscience at Maastricht University.

22. Barker AT, Jalinous R, Freeston IL. Non-invasive magnetic stimulation of human motor cortex. Lancet. (1985) 1:11067. doi: 10.1016/S0140-6736(85)92413-4

23. Pascual-Leone A, Valls-Sole J, Wassermann EM, Hallett M. Responses to rapid-rate transcranial magnetic stimulation of the human motor cortex. Brain: a J Neurol. (1994) 117(Pt 4):847-58. doi: 10.1093/brain/117.4.847

24. George MS, Wassermann EM, Williams WA, Callahan A, Ketter TA, Basser P, et al. Daily repetitive transcranial magnetic stimulation. (rTMS) improves mood in depression. Neuroreport. (1995) 6:1853-6. doi: 10.1097/00001756-199510020-00008

25. Hallett M. Transcranial magnetic stimulation: a primer. Neuron. (2007) 55:187-99. doi: 10.1016/j.neuron.2007.06.026

26. Huang YZ, Edwards MJ, Rounis E, Bhatia KP, Rothwell JC. Theta burst stimulation of the human motor cortex. Neuron. (2005) 45:2016. doi: 10.1016/j.neuron.2004.12.033

27. Suppa A, Huang YZ, Funke K, Ridding MC, Cheeran B, Di Lazzaro $\mathrm{V}$, et al. Ten years of theta burst stimulation in humans: established knowledge, unknowns and prospects. Brain Stimul. (2016) 9:323-35. doi: 10.1016/j.brs.2016.01.006

28. Gamboa OL, Antal A, Moliadze V, Paulus W. Simply longer is not better: reversal of theta burst after-effect with prolonged stimulation. Exp Brain Res. (2010) 204:181-7. doi: 10.1007/s00221-010-2293-4

29. Murakami T, Muller-Dahlhaus F, Lu MK, Ziemann U. Homeostatic metaplasticity of corticospinal excitatory and intracortical inhibitory neural circuits in human motor cortex. J Physiol. (2012) 590:5765-81. doi: 10.1113/jphysiol.2012.238519

30. Tse NY, Goldsworthy MR, Ridding MC, Coxon JP, Fitzgerald PB, Fornito $A$, et al. The effect of stimulation interval on plasticity following repeated blocks of intermittent theta burst stimulation. Sci Rep. (2018) 8:8526. doi: 10.1038/s41598-018-26791-w

31. Opie GM, Vosnakis E, Ridding MC, Ziemann U, Semmler JG. Priming theta burst stimulation enhances motor cortex plasticity in young but not old adults. Brain Stimulation. (2017) 10:298-304. doi: 10.1016/j.brs.2017.01.003

32. Gamboa OL, Antal A, Laczo B, Moliadze V, Nitsche MA, Paulus W. Impact of repetitive theta burst stimulation on motor cortex excitability. Brain Stimul. (2011) 4:145-51. doi: 10.1016/j.brs.2010.09.008

33. Baeken C, Marinazzo D, Wu GR, Van Schuerbeek P, De Mey J, Marchetti I, et al. Accelerated HF-rTMS in treatment-resistant unipolar depression: insights from subgenual anterior cingulate functional connectivity. World J Biol Psychiatry. (2014) 15:286-97. doi: 10.3109/15622975.2013.872295

34. Herremans SC, Van Schuerbeek P, De Raedt R, Matthys F, Buyl R, De Mey J, et al. The impact of accelerated right prefrontal high-frequency repetitive transcranial magnetic stimulation (rTMS) on cue-reactivity: an fMRI study on craving in recently detoxified alcohol-dependent patients. PLoS ONE. (2015) 10:e0136182. doi: 10.1371/journal.pone.0136182

35. Baeken C, Marinazzo D, Everaert H, Wu GR, Van Hove C, Audenaert K, et al. The impact of accelerated HF-rTMS on the subgenual anterior cingulate cortex in refractory unipolar major depression: insights from 18FDG PET brain imaging. Brain Stimul. (2015) 8:808-15. doi: 10.1016/j.brs.2015.01.415

36. McGirr A, Van den Eynde F, Tovar-Perdomo S, Fleck MP, Berlim MT. Effectiveness and acceptability of accelerated repetitive transcranial magnetic stimulation. (rTMS) for treatment-resistant major depressive disorder: an open label trial. J Affect Disord. (2015) 173:216-20. doi: 10.1016/j.jad.2014.10.068

37. Herremans SC, De Raedt R, Van Schuerbeek P, Marinazzo D, Matthys F, De Mey J, et al. Accelerated HF-rTMS protocol has a rate-dependent effect 
on dACC activation in alcohol-dependent patients: an open-label feasibility study. Alcohol Clin Exp Res. (2016) 40:196-205. doi: 10.1111/acer.12937

38. Baeken C. Accelerated rTMS: a potential treatment to alleviate refractory depression. Front Psychol. (2018) 9:2017. doi: 10.3389/fpsyg.2018.02017

39. Blumberger DM, Vila-Rodriguez F, Thorpe KE, Feffer K, Noda Y, Giacobbe P, et al. Effectiveness of theta burst versus high-frequency repetitive transcranial magnetic stimulation in patients with depression. (THREE-D): a randomised non-inferiority trial. Lancet. (2018) 391:168392. doi: 10.1016/S0140-6736(18)30295-2

40. Desmyter S, Duprat R, Baeken C, Van Autreve S, Audenaert K, van Heeringen $\mathrm{K}$. Accelerated intermittent theta burst stimulation for suicide risk in therapyresistant depressed patients: a randomized, sham-controlled trial. Front Human Neurosci. (2016) 10:480. doi: 10.3389/fnhum.2016.00480

41. Duprat R, Desmyter S, Rudi DR, van Heeringen K, Van den Abbeele D, Tandt $\mathrm{H}$, et al. Accelerated intermittent theta burst stimulation treatment in medication-resistant major depression: a fast road to remission? J CAffective Disord. (2016) 200:6-14. doi: 10.1016/j.jad.2016.04.015

42. Thomson AC, de Graaf TA, Kenis G, Rutten BPF, Schuhmann T, Sack AT. No additive meta plasticity effects of accelerated iTBS with short inter-session intervals. Brain Stimul. (2019) 12:1301-3. doi: 10.1016/j.brs.2019.05.012

43. Kramár EA, Babayan AH, Gavin CF, Cox CD, Jafari M, Gall CM, et al. Synaptic evidence for the efficacy of spaced learning. Proc Natl Acad Sci USA. (2012) 109:5121-6. doi: 10.1073/pnas.1120700109

44. Frey U, Schollmeier K, Reymann KG, Seidenbecher T. Asymptotic hippocampal long-term potentiation in rats does not preclude additional potentiation at later phases. Neuroscience. (1995) 67:799-807. doi: 10.1016/0306-4522(95)00117-2

45. Cao G, Harris KM. Augmenting saturated LTP by broadly spaced episodes of theta-burst stimulation in hippocampal area CA1 of adult rats and mice. $J$ Neurophysiol. (2014) 112:1916-24. doi: 10.1152/jn.00297.2014

46. Larson J, Lynch G. Induction of synaptic potentiation in hippocampus by patterned stimulation involves two events. Science. (1986) 232:9858. doi: $10.1126 /$ science. 3704635

47. Larson J, Munkácsy E. Theta-burst LTP. Brain Res. (2015) 1621:3850. doi: 10.1016/j.brainres.2014.10.034

48. Lynch G, Kramar EA, Babayan AH, Rumbaugh G, Gall CM. Differences between synaptic plasticity thresholds result in new timing rules for maximizing long-term potentiation. Neuropharmacology. (2013) 64:2736. doi: 10.1016/j.neuropharm.2012.07.006

49. Karabanov A, Ziemann U, Hamada M, George MS, Quartarone A, Classen J, et al. Consensus paper: probing homeostatic plasticity of human cortex with non-invasive transcranial brain stimulation. Brain Stimul. (2015) 8:44254. doi: 10.1016/j.brs.2015.01.404

50. Williams NR, Sudheimer KD, Bentzley BS, Pannu J, Stimpson KH, Duvio D, et al. High-dose spaced theta-burst TMS as a rapid-acting antidepressant in highly refractory depression. Brain. (2018) 141:e18e. doi: 10.1093/brain/awx379

51. Hensel L, Grefkes C, Tscherpel C, Ringmaier C, Kraus D, Hamacher S, et al. Intermittent theta burst stimulation applied during early rehabilitation after stroke: study protocol for a randomised controlled trial. BMJ Open. (2019) 9:e034088. doi: 10.1136/bmjopen-2019-034088

52. Korzhova J, Bakulin I, Sinitsyn D, Poydasheva A, Suponeva N, Zakharova $\mathrm{M}$, et al. High-frequency repetitive transcranial magnetic stimulation and intermittent theta-burst stimulation for spasticity management in secondary progressive multiple sclerosis. Eur J Neurol. (2019) 26:680e44. doi: 10.1111/ene.13877

53. Naro A, Billeri L, Cannavo A, De Luca R, Portaro S, Bramanti $P$, et al. Theta burst stimulation for the treatment of obsessivecompulsive disorder: a pilot study. J Neural Transm. (2019) 126:1667-77. doi: 10.1007/s00702-019-02098-6

54. Rossi S, Hallett M, Rossini PM, Pascual-Leone A. Safety, ethical considerations, and application guidelines for the use of transcranial magnetic stimulation in clinical practice and research. Clin Neurophysiol. (2009) 120:2008-39. doi: 10.1016/j.clinph.2009. 08.016

55. Lefaucheur JP, Andre-Obadia N, Antal A, Ayache SS, Baeken C, Benninger $\mathrm{DH}$, et al. Evidence-based guidelines on the therapeutic use of repetitive transcranial magnetic stimulation. (rTMS). Clin Neurophysiol. (2014) 125:2150-206. doi: 10.1016/j.clinph.2014.05.021
56. Lefaucheur J-P, Aleman A, Baeken C, Benninger DH, Brunelin J, Di Lazzaro $\mathrm{V}$, et al. Evidence-based guidelines on the therapeutic use of repetitive transcranial magnetic stimulation. (rTMS): an update. (2014-2018). Clin Neurophysiol. (2020) 131:474-528. doi: 10.1016/j.clinph.2020.02.003

57. Weiler M, Stieger KC, Long JM, Rapp PR. Transcranial magnetic stimulation in alzheimer's disease: are we ready? eNeuro. (2020) 7:ENEURO.023519.2019. doi: 10.1523/ENEURO.0235-19.2019

58. Cotelli M, Calabria M, Manenti R, Rosini S, Zanetti O, Cappa $\mathrm{SF}$, et al. Improved language performance in Alzheimer disease following brain stimulation. J Neurol Neurosurg Psychiatry. (2011) 82:794-7. doi: 10.1136/jnnp.2009.197848

59. Del Giacco L, Pistocchi A, Cotelli F, Fortunato AE, Sordino P. A peek inside the neurosecretory brain through Orthopedia lenses. Dev Dyn. (2008) 237:2295-303. doi: 10.1002/dvdy.21668

60. Cotelli M, Manenti R, Cappa SF, Geroldi C, Zanetti O, Rossini $\mathrm{PM}$, et al. Effect of transcranial magnetic stimulation on action naming in patients with Alzheimer disease. Arch Neurol. (2006) 63:1602-4. doi: 10.1001/archneur.63.11.1602

61. Rutherford G, Gole R, Moussavi Z. rTMS as a treatment of alzheimer's disease with and without comorbidity of depression: a review. Neurosci J. (2013) 2013:679389. doi: 10.1155/2013/679389

62. Kim TD, Hong G, Kim J, Yoon S. Cognitive enhancement in neurological and psychiatric disorders using transcranial magnetic stimulation (TMS): a review of modalities, potential mechanisms and future implications. Exp Neurobiol. (2019) 28:1-16. doi: 10.5607/en.2019.28.1.1

63. Mariorenzi R, Zarola F, Caramia MD, Paradiso C, Rossini PM. Non-invasive evaluation of central motor tract excitability changes following peripheral nerve stimulation in healthy humans. Electroencephalogr Clin Neurophysiol. (1991) 81:90-101. doi: 10.1016/0168-5597(91)90002-F

64. Stefan K, Kunesch E, Cohen LG, Benecke R, Classen J. Induction of plasticity in the human motor cortex by paired associative stimulation. Brain. (2000) 123(Pt 3):572-84. doi: 10.1093/brain/123.3.572

65. Ziemann U. LTP-like plasticity in human motor cortex. Suppl Clin Neurophysiol. (2004) 57:702-7. doi: 10.1016/S1567-424X(09)70410-6

66. Muller JF, Orekhov Y, Liu Y, Ziemann U. Homeostatic plasticity in human motor cortex demonstrated by two consecutive sessions of paired associative stimulation. Eur J Neurosci. (2007) 25:3461-8. doi: 10.1111/j.1460-9568.2007.05603.x

67. Jung P, Ziemann U. Homeostatic and nonhomeostatic modulation of learning in human motor cortex. J Neurosci. (2009) 29:5597604. doi: 10.1523/JNEUROSCI.0222-09.2009

68. Rossini PM, Di Iorio R, Bentivoglio M, Bertini G, Ferreri F, Gerloff C, et al. Methods for analysis of brain connectivity: An IFCN-sponsored review. Clin Neurophysiol. (2019) 130:1833-58. doi: 10.1016/j.clinph.2019.06.006

69. Hallett M, de Haan W, Deco G, Dengler R, Di Iorio R, Gallea C, et al. Human brain connectivity: Clinical applications for clinical neurophysiology. Clin Neurophysiol. (2020) 131:1621-51. doi: 10.1016/j.clinph.2020.03.031

70. Ferbert A, Priori A, Rothwell JC, Day BL, Colebatch JG, Marsden CD. Interhemispheric inhibition of the human motor cortex. J Physiol. (1992) 453:525-46. doi: 10.1113/jphysiol.1992.sp019243

71. Fox MD, Buckner RL, White MP, Greicius MD, Pascual-Leone A. Efficacy of TMS targets for depression is related to intrinsic functional connectivity with the subgenual cingulate. Biol Psychiatr. (2012) 72:595603. doi: 10.1016/j.biopsych.2012.04.028

72. Liston C, Chen AC, Zebley BD, Drysdale AT, Gordon R, Leuchter $B$, et al. Default mode network mechanisms of transcranial magnetic stimulation in depression. Biol Psychiatr. (2014) 76:517-26. doi: 10.1016/j.biopsych.2014.01.023

Conflict of Interest: The authors declare that the research was conducted in the absence of any commercial or financial relationships that could be construed as a potential conflict of interest.

Copyright $\odot 2020$ Thomson and Sack. This is an open-access article distributed under the terms of the Creative Commons Attribution License (CC BY). The use, distribution or reproduction in other forums is permitted, provided the original author(s) and the copyright owner(s) are credited and that the original publication in this journal is cited, in accordance with accepted academic practice. No use, distribution or reproduction is permitted which does not comply with these terms. 